\title{
Overcoming the Efficiency Limitation of Low Microwave Power Harvesting with Backward Tunnel Diodes
}

\author{
Carlos H. P. Lorenz ${ }^{1}$, Student Member IEEE, Simon Hemour ${ }^{1}$, Member IEEE, Wenjun Li ${ }^{2}$, Yi Xie ${ }^{2}$, Jules \\ Gauthier ${ }^{1}$, Member, IEEE, Patrick Fay ${ }^{2}$, Senior Member IEEE, Ke Wu ${ }^{1}$, Fellow, IEEE \\ ${ }^{1}$ Poly-Grames, École Polytechnique de Montréal, Montreal, QC H3T 1J4, Canada \\ ${ }^{2}$ Dept. of Electrical Engineering, Univ. of Notre Dame, Notre Dame, IN 46556, USA
}

\begin{abstract}
This paper explores, for the first time, the use of high responsivity heterostructure backward tunnel diodes to enhance the conversion efficiency of ambient microwave power harvesters. Progress in advancing the performance of low power rectifiers has been slowed because the maximum possible efficiency using Schottky diodes has been reached. Measurements of RF-to-DC conversion efficiency at $-40 \mathrm{dBm} / 2.4 \mathrm{GHz}$ are reported in this paper, showing that the backward diode outperforms the HSMS-285B Schottky diode by a factor of $\mathbf{1 0 . 5}$ and the Skyworks SMS 7630 by a factor of 5.5. A narrowband rectifier circuit was designed, fabricated and tested, showing a total efficiency of $3.8 \%$ for a $100 \mathrm{nW}$ input RF power and $18.2 \%$ at $1 \mu \mathrm{W}$ input $\mathrm{RF}$ power, at $2.35 \mathrm{GHz}$.
\end{abstract}

Index Terms - Backward tunnel diode, Schottky diode, microwave power harvesting, microwave power rectification, rectenna.

\section{INTRODUCTION}

Emerging technologies and applications such as the Internet of Things (IoT), Smart Cities, Building Automation \& Monitoring and Wearable Devices usually require physically small power sources to remotely power those embedded sensing and controlling devices. Microwave Power Harvesting (MPH) has been proposed as an alternative wireless power source for such low power consumption devices [1-3].

However, an overview of the state of the art suggests that a limitation in the efficiency of low power microwave rectifiers, based on Schottky diodes, has been reached [2, 4, 5]. Microwave power rectifiers capable of operating below -30 $\mathrm{dBm}$ are needed for ambient MPH $[1,6]$; however to date, RF-DC conversion efficiency has not yet reached $10 \%$ at frequencies above $1 \mathrm{GHz}$ at such low power levels. Overcoming this performance barrier is essential for MPH systems to become a practical reality.

Schottky diodes rely on the thermionic effect, which limits their zero-bias current responsivity, $\mathfrak{R}_{I 0}$, to $19.34 \mathrm{~A} / \mathrm{W}$ at $300 \mathrm{~K}$ [4]. According to $[4,5]$, the rectification efficiency of microwave rectifiers operating in the diode's square-law region is directly proportional to the square of $\mathfrak{R}_{I 0}$. Recently, backward diodes, which make use of quantum mechanical tunneling rather than thermionic emission, were reported to overcome the Schottky diodes' $\mathfrak{R}_{I 0}$, reaching values near 24 A/W $[4,7]$. This paper explores the application of such diodes to overcome the RF-DC conversion efficiency limitation seen in Schottky diode microwave rectifiers. A comparison between a backward diode with zero-bias junction resistance $R_{j 0}$ near $10 \mathrm{k} \Omega$, and an HSMS-285B schottky diode is presented, putting in evidence the increase in extremely low input power RF-DC conversion efficiency, namely ambient power below $-30 \mathrm{dBm}$.

\section{TUNNEL DIODE CHARACTERIZATION}

On-wafer characterization has been done using $100 \mu \mathrm{m}$ pitch GSG probe, on a Cascade Summit probe station. A Keysight PNA network analyzer was used to retrieve the [S11] parameter, while the diode sensitivity and the I(V) characteristics were retrieved using the internal bias-T of the VNA and measured using a 6-1/2 digit HP 34401A voltmeter. The DC current-voltage characteristic, as well as the diode's sensitivity and reflection coefficient $\Gamma$ from $500 \mathrm{MHz}$ to $40 \mathrm{GHz}$ were measured for an input power ranging from -30 $\mathrm{dBm}$ to $-5 \mathrm{dBm}$, in $5 \mathrm{dBm}$ steps.

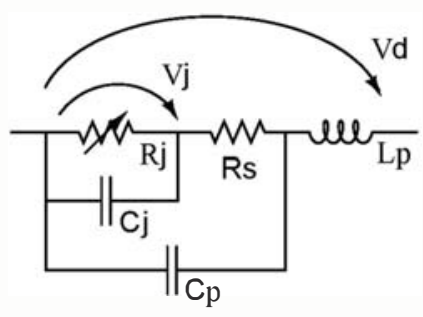

Fig. 1. Shockley diode model with package/pads parasitic components, used in the modeling of the measured backward diode. $R_{j}$ represents the diode's non-linear junction resistance, $C_{j}$ the junction capacitance, $C_{p}$ the pad parasitic capacitance, $L_{p}$, the pad inductance and $R_{S}$ the series resistance.

The diode model parameters in Fig. 1 were extracted from the measurements. The nonlinear junction current-voltage equation $\mathrm{Ij}(\mathrm{Vj})$ was modeled using a $7^{\text {th }}$ order polynomial (1). The comparison between the modeling results and measurements is given in Fig. 2. The DC non-linear model has been verified between $-100 \mathrm{mV}$ and $200 \mathrm{mV}$, enough range to cover the ambient energy harvesting applications.

$$
\begin{aligned}
& I_{j}\left(V_{j}\right)=7.99 \cdot 10^{-5}\left(V_{j}\right)-1.73 \cdot 10^{-3}\left(V_{j}^{2}\right)+1.27 \cdot 10^{-2}\left(V_{j}^{3}\right)+ \\
& 6.76 \cdot 10^{-3}\left(V_{j}^{4}\right)-0.58\left(V_{j}^{5}\right)+2.92\left(V_{j}^{6}\right)-4.65\left(V_{j}^{7}\right)
\end{aligned}
$$




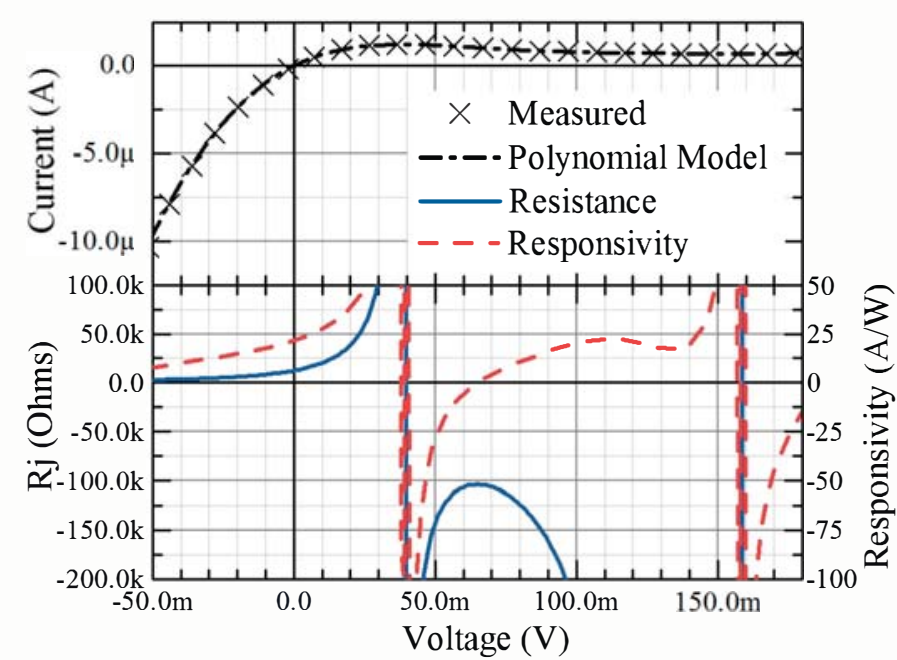

Fig. 2. Measured and modeled backward tunnel diode $I-V$ curve, together with the calculated value of the diode's $R_{j}$ and $\Re$.

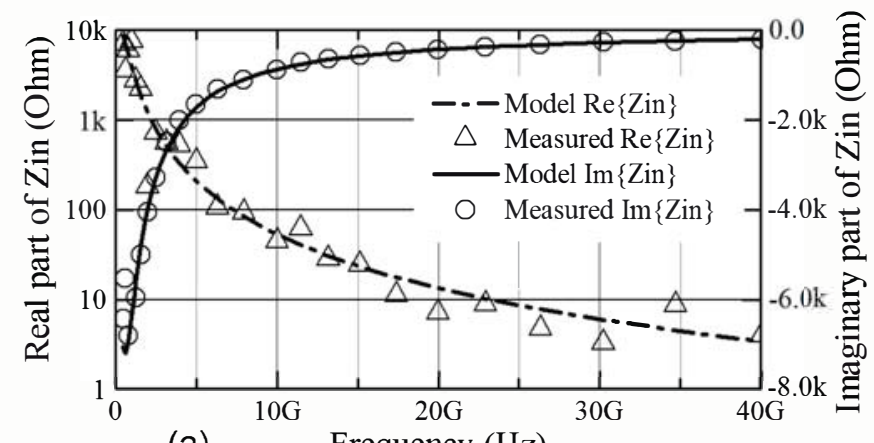

(a)

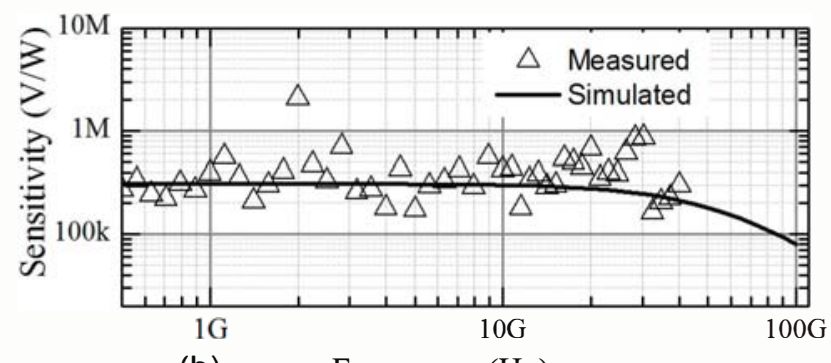

(b)

Frequency $(\mathrm{Hz})$

Fig. 3. Comparison between measured and modeled backward diode RF Characteristics at $-30 \mathrm{dBm}$. (a) Input impedance, $Z_{\text {in, }}$, real and imaginary components. (b) Voltage sensitivity; the simulation has been projected to $100 \mathrm{GHz}$.

Using the above-described $I j(V j)$ polynomial, the diode's junction resistance $R_{j}$, and current responsivity $\Re_{I}$ were calculated [8]. Having the non-linear junction behavior already defined, the measured sensitivity and reflection coefficient were then used to extract the linear parasitic model parameters; which were found to be $C_{p}=13.7 \mathrm{fF}, R_{s}=25 \Omega$ and $L_{p}=52.6 \mathrm{pH}$. Although $\mathrm{C}_{\mathrm{j}}$ may in general be bias dependent, for the model developed here $C_{j}$ was approximated by a linear capacitance of $4.5 \mathrm{fF}$. Modeling results and measurements are compared in Fig. 3. The simulation results were projected up to $100 \mathrm{GHz}$.

\section{THE MICROWAVE RECTIFIER}

According to the small signal theory, a microwave rectifier efficiency, when connected to the optimum DC load, can be approximated by (2) when operating near the square-law region $[4,5]$ :

$$
\eta_{R F D C}=\frac{P_{D C}}{P_{R F}}=\left(P_{R F} \frac{\Re_{I 0}^{2} \cdot R_{j 0}}{4}\right) \cdot\left(\eta_{p}\right)
$$

where $\eta_{p}$ is the diode's parasitic efficiency and $P_{R F}$ is the power delivered to the diode. Disregarding matching and parasitic losses, the studied backward diode has a calculated RF to DC conversion efficiency equal to $14.7 \%$ at $-40 \mathrm{dBm}$, while an optimum ideal Schottky diode with $\Re_{I 0}=19.34 \mathrm{~A} / \mathrm{W}$ and the same $R_{j 0}=12.5 \mathrm{k} \Omega$ as the studied backward diode, would present an efficiency equal to $11.7 \%$. Comparing it to the HSMS-285B, which is a Schottky diode widely used in energy harvesting studies, and has $R_{j 0}=9.1 \mathrm{k} \Omega$ and $\Re_{I 0}=18.25$ $\mathrm{A} / \mathrm{W}$, the expected efficiency disregarding matching and parasitic losses would be $7.6 \%$ (See Table 1).

In order to verify the increase in efficiency predicted by the theory, both HSMS-285B and the backward diode rectification efficiencies were measured. The choice of the Schottky diode was based on the value of $R_{j 0}$, which should be near to that presented by the backward diode to ensure a valid comparison.

The microwave power rectification setup consisted of a Keysight PNA Network Analyzer with an integrated bias-T, a Focus microwave tuner system used as matching network, and a 6-1/2 digit HP 34401A multimeter connected to a variable resistor, used as load. The output power of the PNA was calibrated at the tuner input (source side); so it could be used as the microwave power source. At the same time the PNA could be used to measure the reflections to ensure that good matching was obtained at $2.4 \mathrm{GHz}$. A schematic diagram of the setup is shown in Fig. 4.

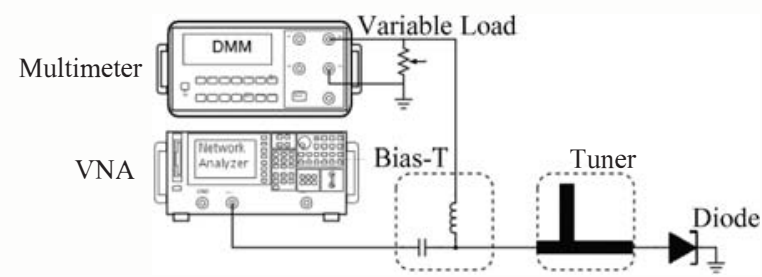

Fig. 4. Simplified schematic of the setup used for the microwave rectifier RF-DC efficiency characterization.

To evaluate the power incident on the diodes, the opencircuit detector voltage was measured, using the diode as a microwave power detector. This information together with the diode's sensitivity measured during the characterization step was then used to extract the insertion losses of the matching circuit. In this way, the rectifier's RF-DC conversion efficiency $\eta_{R F-D C}$ and parasitic efficiency $\eta_{\mathrm{P}}$ were evaluated for both diode types, independently of any losses that originated from the circuit implementation. 
For the efficiency measurement, the variable load was adjusted to its optimum value, $13 \mathrm{k} \Omega$ for the backward diode, and $9 \mathrm{k} \Omega$ for the HSMS-285B diode. The results are given in Fig. 5 together with an indication of the maximum efficiency that an ideal Schottky diode with no parasitic losses and the same $R_{j 0}$ as the studied backward diode could attain.

Fig. 5 shows two advantages of the evaluated backward diode for energy harvesting applications. The first is the improvement in power conversion efficiency due to the higher responsivity. Even when compared to the ideal Schottky diode theoretical limit, the backward diode has a $25.3 \%$ higher efficiency at extremely low input power. The second advantage is due to the low junction capacitance resulting from the backward diode construction. The resulting parasitic loss is extremely low at $2.4 \mathrm{GHz}$, leading to a 10.5 times higher efficiency when compared to the HSMS-285B diode.

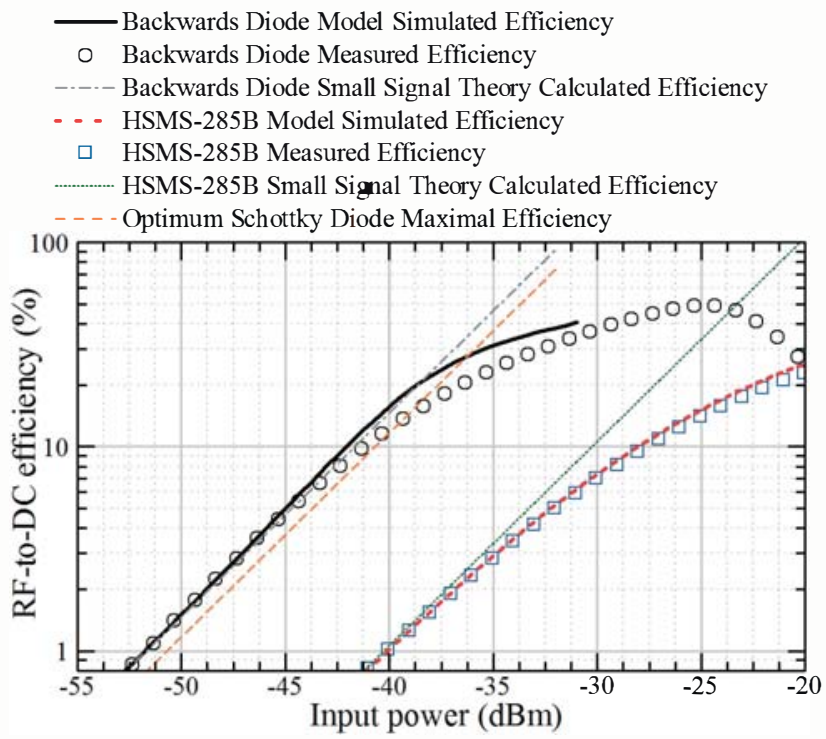

Fig. 5. Measured, simulated and calculated efficiencies of the backward tunnel diode and HSMS-285B diode microwave rectifiers at $2.4 \mathrm{GHz}$. Matching network losses have been de-embedded.

TABLE I

RECTIFIER EFFICIENCIES AT -40dBm AND 2.4 GHz, USING (1)

\begin{tabular}{|l|c|c|c|c|c|c|}
\hline \multicolumn{1}{|c|}{ Diode } & \multirow{2}{*}{$\begin{array}{c}R_{10} \\
\end{array}$} & \multirow{2}{*}{$\begin{array}{l}R_{j 0} \\
\mathrm{k} / \mathrm{W})\end{array}$} & \multirow{2}{*}{$\begin{array}{c}C_{j 0} \\
\mathrm{fF}\end{array}$} & \multicolumn{3}{|c|}{ Efficiency (\%) } \\
\cline { 5 - 8 } & & $\eta_{R F-D C}$ & $\eta_{P}$ & Total \\
\hline Backward & 21.65 & 12.5 & 4.5 & $\mathbf{1 4 . 7}$ & 99.7 & 14.6 \\
\hline $\begin{array}{l}\text { Ideal } \\
\text { Schottky }\end{array}$ & 19.34 & 12.5 & $\mathrm{NA}$ & $\mathbf{1 1 . 7}$ & $\mathrm{NA}$ & $\mathrm{NA}$ \\
\hline HSMS285B & 18.25 & 9.13 & 180 & $\mathbf{7 . 6}$ & 13.9 & 1.1 \\
\hline SMS7630 & 18.42 & 5.43 & 140 & $\mathbf{4 . 6}$ & 45.4 & 2.1 \\
\hline
\end{tabular}

Table 1 shows the RF-to-DC and parasitic efficiencies [4] calculated using the backward diode model parameters and Schottky diode's datasheet parameters. The SMS7630 Schottky diode was added to this comparison as it is another largely used diode in today's MPH applications. The calculated efficiency for the backward diode is 5.5 times higher than the scenario with the SMS7630 diode. As can be seen in Table 1, both the intrinsic RF-DC efficiency and parasitic efficiency are improved with the use of a backward diode compared to Schottky diodes.

A narrow-band rectifier has been fabricated to demonstrate the operation in a more realistic system context. The first version of this circuit exhibited high matching network insertion losses and a shift at operating frequency (from 2.4 $\mathrm{GHz}$ to $2.35 \mathrm{GHz}$ ) because of the use of an in-house fabricated GSG probe (rather than wire-bonding). Despite this additional loss, an overall rectifier efficiency at $100 \mathrm{nW}$ of $3.8 \%$ (Fig. 6b) has been measured. Although well below the $11.6 \%$ expected from the measurements in Fig. 5, the device technology appears promising based on this first demonstration, remaining above the state-of-the-art Schottky diode rectifiers results.
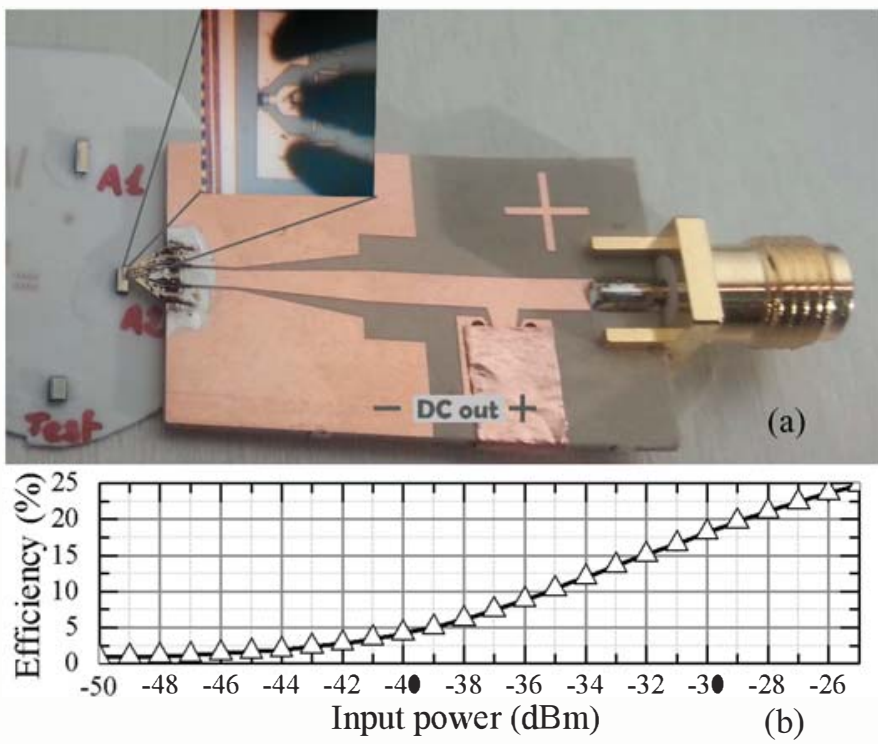

Fig. 6. (a) Photograph of the rectifier circuit using a backward diode. An in-house GSG probe is used to interface with the diode pads (inset). (b) Measured efficiency versus input power at 2.35 $\mathrm{GHz}$

\section{CONCLUSION}

This paper has reported for the first time the possibility of overcoming the microwave power rectification efficiency limits of Schottky diode rectifiers at extremely low power levels, through the use of backward tunnel diodes [7]. A $25.3 \%$ increase in efficiency was observed when the backward diode rectifier was compared to a theoretically ideal Schottky diode with the same $R_{j 0}$, while a 10.5 times higher efficiency was obtained when compared to a real HSMS-285B Schottky diode, due to lower diode parasitic losses and improved intrinsic responsivity. 


\section{ACKNOWLEDGEMENT}

This work was supported in part by the Natural Sciences and Engineering Research Council of Canada (NSERC) and CREATE PERSWADE Training Program.

The authors would like to thank the Poly-GRAMES technicians for their valuable experience and support during fabrication and experiments set-up.

\section{REFERENCES}

[1] K. Sangkil, R. Vyas, J. Bito, et al., "Ambient RF Energy-Harvesting Technologies for Self-Sustainable Standalone Wireless Sensor Platforms," Proceedings of the IEEE, vol. 102, pp. 1649-1666, 2014.

[2] Z. Popovic, "Cut the cord: Low-power far-field wireless powering," Microwave Magazine, IEEE, vol. 14, pp. 55-62, 2013.

[3] D. Masotti, A. Costanzo, P. Francia, et al., "A LoadModulated Rectifier for RF Micropower Harvesting With Start-Up Strategies," Microwave Theory and Techniques, IEEE Transactions on, vol. 62, pp. 9941004, 2014.

[4] S. Hemour and K. Wu, "Radio-Frequency Rectifier for Electromagnetic Energy Harvesting: Development Path and Future Outlook," Proceedings of the Ieee, vol. 102, pp. 1667-1691, Nov 2014.

[5] S. Hemour, Y. P. Zhao, C. H. P. Lorenz, et al., "Towards Low-Power High-Efficiency RF and Microwave Energy Harvesting," Ieee Transactions on Microwave Theory and Techniques, vol. 62, pp. 965-976, Apr 2014.

[6] Paul D. Mitcheson, et al. (2014, June 14). London RF Survey. Available: http://www.londonrfsurvey.org/

[7] Z. Zhang, R. Rajavel, P. Deelman, and P. Fay, "SubMicron Area Heterojunction Backward Diode Millimeter-Wave Detectors With 0.18 Noise Equivalent Power," Microwave and Wireless Components Letters, IEEE, vol. 21, pp. 267-269, 2011.

[8] H. C. Torrey, C. A. Whitmer, and S. A. Goudsmit, Crystal rectifiers, 1st ed. New York, : McGraw-Hill Book Co., 1948. 